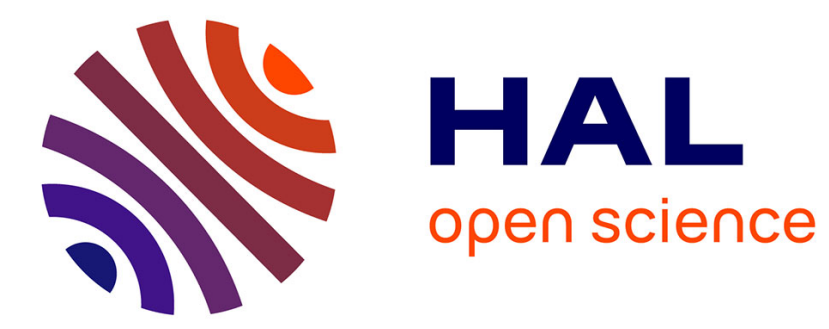

\title{
A finite element method applied to new active contour models and 3D reconstruction from cross sections
}

\author{
Laurent D. Cohen, Isaac Cohen
}

\section{To cite this version:}

Laurent D. Cohen, Isaac Cohen. A finite element method applied to new active contour models and 3D reconstruction from cross sections. [Research Report] RR-1245, INRIA. 1990. inria-00075313

\section{HAL Id: inria-00075313 \\ https://hal.inria.fr/inria-00075313}

Submitted on 24 May 2006

HAL is a multi-disciplinary open access archive for the deposit and dissemination of scientific research documents, whether they are published or not. The documents may come from teaching and research institutions in France or abroad, or from public or private research centers.
L'archive ouverte pluridisciplinaire HAL, est destinée au dépôt et à la diffusion de documents scientifiques de niveau recherche, publiés ou non, émanant des établissements d'enseignement et de recherche français ou étrangers, des laboratoires publics ou privés. 


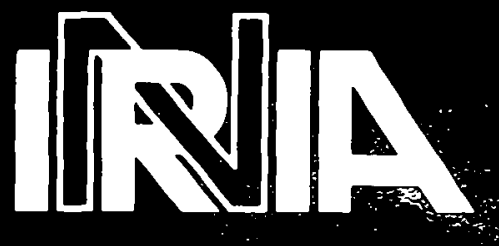

UNITÉ DE RECHERCHE INRIA-ROCOUENEOURT

\title{
Rapports de Recherche
}

\author{
$N^{\circ} 1245$ \\ Programme 6 \\ Robotique, Image et Vision
}

\section{A FINITE ELEMENT METHOD APPLIED TO NEW ACTIVE CONTOUR MODELS AND 3D RECONSTRUCTION FROM CROSS SECTIONS}

Institut National de Recherche en Informatique et en Automatique

Laurent D. COHEN Isaac COHEN

Domaine de Voluceau

Rocquencourt B.P.105

78153 LeGhesnay Cedex

France

Juin 1990

Tél.:(1)39635511 


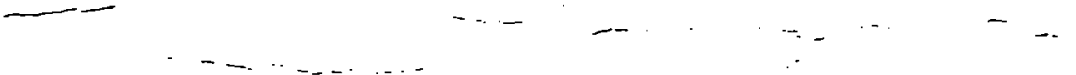




\title{
A finite element method applied to new active contour models and 3D reconstruction from cross sections
}

\section{Utilisation des élèments finis pour un nouveau modèle de contours actifs et reconstruction 3D à partir de coupes successives.}

\author{
Laurent D. COHEN \\ CEREMADE, U.R.A. CNRS 749, Université Paris IX - Dauphine \\ Place du Marechal de Lattre de Tassigny 75775 Paris CEDEX, France.
}

\section{Isaac COHEN}

INRIA, Domaine de Voluceau, Rocquencourt B.P. 105, 78153 Le Chesnay CEDEX, France. 
example of figure 7 .

3. We replace the finite difference method of $[9,12]$ by a finite element method (FEM). With finite differences, we only have a discrete knowledge of the functions at some points of subdivision and have no information between these points. Therefore the distance between successive points must remain very small not to miss too much information, since the external forces are applied to grid of points. This typically yields large linear systems to solve. Conversely, with the FEM, we are really working on continuous curves whatever the size of the used grid. Therefore, the studied curve is known everywhere in the image, independently of the chosen discretization. This yields a lower algorithmic complexity and better numerical stability.

After recalling the basic principles of "snakes" and "balloons", we give details about our model and the numerical solution of the evolution equation by finite'elements in space and finite difference in time. We illustrate our technique by showing the automatic segmentation of medical images. Finally, we give the first $3 \mathrm{D}$ reconstruction results obtained by propagating the segmentation in a series of successive slices.

\section{Energy minimizing curves and active contours model}

The active contours (called Snakes) are a special case of deformable models as presented in $\{11\}$. The contour model is a mapping :

$$
\begin{gathered}
\Omega=[0,1] \rightarrow \Re^{2} \\
\left.s \mapsto v(s)=\left(x(s), y^{\prime} s\right)\right)
\end{gathered}
$$




\section{Introduction}

We introduce a new model for active contours, which significantly improves the detection quality of closed edges, enhances numerical stability while reducing computational complexity. Our model was used to segment automatically noisy ultrasound and Magnetic Resonance images of the beating heart, both in 2 and 3 dimensions. We present the features of this new model, with a number of various significant experimental results, and we discuss future research. A generalization of the method to introduce general $3 \mathrm{D}$ deformable models is presented in a companion paper.

We consider the application of deformable contour models [6] to extract smooth shapes in a given region of the image. The philosophy of the approach is to introduce an elastic curve in the image, and let it evolve from an initial position under the action of both internal forces (elasticity parameters of the curve) and external forces (attraction towards local edgels). If this original idea is due to $\{3,9,12\}$, our model presents the following interesting new features:

1. We take into account edge points previously extracted by a local edge detector. This allows to combine the quality of a good local edge detector, e.g. a Canny-Deriche edge extractor $[4, \tau, 10]$, with a global active model.

2. We introduce an internal pressure force by considering our curve as a balloon which is inflated. We add to the previous internal and external forces a pressure force pushing out of the curve as if we were introducing air inside. The curve expands and is attracted by edges as before. But if the edge is too small or too weak with respect to the pressure force, the curve just passes through.

This avoids the curve being "trapped" by spurious isolated edge points, and makes the result much more insensitive to the initial conditions. For instance, one can choose as an initial condition almost any simple closed curve within the interior of the considered region in the 


\begin{abstract}
The use of energy-minimizing curves, known as "snakes" to extract features of interest in images has been introduced by Kass, Witkin \& Terzopoulos. We present a model of deformation which solves some of the problems encountered with the original method such as instability and initial data while reducing the computational complexity.

This model makes the curve behave like a balloon which is inflated by an additional force. The initial curve need no longer be close to the solution to converge. The external forces, that push the curve to the edges, are modified to give more stable results.

The system is solved using a conform finite element method in the minimization process. The evolution to the equilibrium presents less oscillations, convergence obtained faster and the final results are more accurate.

We have applied this model for segmenting ultrasound and Magnetic resonance images. We have also made a first stage to $3 \mathrm{D}$ object reconstruction, by tracking the extracted contour on a series of successive cross sections.
\end{abstract}

\title{
Resumé
}

Nous présentons un modèle de contours actifs permettant d'extraire les attributs d'une image. Cette méthode de segmentation a été introduite par Kass, Witkin \& Terzepoulos sous le nom de "snake". Le modèle presenté permet de résoudre certains problèmes rencontrés avec la méthode originale tels que l'instabilité et la donnée d'une condition initiale, tout en reduisant la complexité algorithmique.

Dans ce modèle la courbe peut se comporter comme un ballon qu'on gonfle en y ajoutant une force supplementaire. Dans ce cas il y a convergence de la courbe vers la solution sans que la condition initiale soit dans un proche voisinnage de cette solution. Le champs de forces exterieures permettant à la courbe d'être attirée par les contours est également modifié afin d'obtenir des résultats plus stables.

La résolution des équations obtenues en minimisant l'énergie, sont résolus à l'aide d'une méthode d'élements finis. Ainsi l'évolution de la courbe vers un equilibre est plus rapide et la solution obtenue à l'equilibre est plus précise.

Nous avons utilisé ce modéle pour segmenter des images échographiques et à resonance magnétique. Nous avons également réalisé des reconstructions tridimensionnelles en propageant les contours extraits sur une succession de coupes $2 \mathrm{D}$. 
A deformable model, is defined by a space of admissible deformations $A d^{\prime}$ and a functional $E$ to minimize. This functional represents the energy of the model and has the following form:

$$
\begin{gathered}
E: A d \rightarrow \Re \\
v \mapsto E(v)=\int_{\Omega} w_{1}\left|v^{\prime}\right|^{2}+w_{2}\left|v^{\prime \prime}\right|^{2}+P(v) d s
\end{gathered}
$$

where $P$ is the potential associated to the external forces. The potential depends on the image data, and is computed in different ways according to the aimed goal. So if we want the snake to be attracted by edge points, the potential has to depend on the gradient of the image. The set of admissible deformations $A d$ can be restricted by differents boundary conditions; fixed boundary points (ie. $v(0)$ and $v(1)$ are given), cyclic conditions (ie. $v(0)=v(1)$ ) or other types of boundary conditions.

The mechanical properties of the model are defined by the functions $w_{j}$. Their choice determines the elasticity and rigidity of the model.

The energy can be written as the sum of two terms:

$$
E=E_{\text {int }}+E_{\text {image }}
$$

If $v$ is a local minimum for $E$, it satisfies the associated Euler-Lagrange equation:

$$
\left\{\begin{array}{l}
-\left(w_{1} v^{\prime}\right)^{\prime}+\left(w_{2} v^{\prime \prime}\right)^{\prime \prime}+\nabla P(v)=0 \\
+ \text { Boundary Conditions }
\end{array}\right.
$$

which represents the necessary condition for the minimum as $E^{\prime}(v)=0$. In this formulation each term represents a force applied to the curve. A solution can be seen either as realizing the equilibrium of the forces in the equation or reaching the minimum of its energy.

Thus the curve is under the control of two forces:

- Ent represents internal forces which impose the regularity of the curve. $u_{1}$ and $u_{2}$ impose the elasticity and rigidity of the curve. 
- $E_{\text {image }}$ pushes the curve to the significant lines which correspond to the desired attributes. This energy is defined by the potential

$$
P(v)=-|\nabla I(v)|^{2}
$$

The curve is then attracted by the local minima of the potential, which means the local maxima of the gradient, that is contours (see [8] for a more complete discussion about the relation between minimizing the energy and locating contours).

Since the energy is not convex, there are many local minima of $E$. But we are interested in finding a good contour in a given area. We suppose in fact that we have a rough prior estimation of the curve. This estimation is used to solve the associated evolution equation:

$$
\left\{\begin{array}{l}
\frac{\partial v}{\partial t}-\left(w_{1} v^{\prime}\right)^{\prime}+\left(w_{2} v^{\prime \prime}\right)^{\prime \prime}=F(v) \\
v(0, s)=v_{0}(s) \text { initial estimation } \\
+ \text { Boundary Conditions }
\end{array}\right.
$$

where $F$ is the sum of the external forces applied to the curve. A solution of the static problem is found when the previous solution $v(t)$ stabilizes. Then the term $\frac{\partial v}{\partial t}$ tends to 0 thus providing a solution to the static problem.

\section{Improving the model}

Solving the problem as set in the previous section leads to two difficulties we solve in this section. In both cases we give a new definition of the forces, focusing on the evolution equation formulation even though the forces no longer derive from a potential.

To describe these problems, we must start with the discrete form of the equation (2). The simplest way for making this, is the use of the finite difference method both in space and time. 


\subsection{Finite differences Solution}

The evolution problem becomes after finite differences in time (step $\tau$ ) and space (step $h$ ):

$$
v^{t}=(I d+\tau A)^{-1}\left(v^{t-1}+\tau F\left(v^{t-1}\right)\right)
$$

where $A$ is a pentadiagonal banded symmetric positive matrix. Its coefficients depend on the values of $v, w_{1}, w_{2}$ at points $i h$. Thus, we obtain a linear system and we have to inverse a pentadiagonal banded symmetric positive matrix (details can be found in $! 6 j$ ).

\subsection{Instability due to image forces}

Let us examine the effect of the image force $F=-\nabla P$ as defined in the previous section. The direction of $F$ is the steepest descent for $P$, which is natural since we want to get a minimum of $P$ and equilibrium is achieved at points where $P$ is a minimum in the direction normal to the curve. However, due to the discretization of the evolution problem, even though the initial guess can be close to an edge, instabilities can occur. We see from equation (3) that position at time $t, v^{t}$ is obtained after a displacement of $v^{t-1}$ along vector $\tau F\left(v^{t-1}\right)$ and then an inversion of the linear system. This leads to the following remarks

- Time discretization: if $\tau F\left(v^{t-1}\right)$ is too large, point $v^{t-1}$ can cross away the wished minimum and never come back (see figure 1). So either the curve make large oscillations without reaching equilibrium or reaches a different minimum

If we choose $\tau$ small enough such that displacements $\tau F\left(v^{t-1}\right)$ are never too large, for example never larger than one pixel. then small values of $F$ will not have enough effect on the curve and only very few points with high gradient will attract the curve. So instead of acting on time step, we modify the force by normalizing it, taking $F=-k \frac{\nabla P}{\nabla P}$. where $r k$ is of the order of one pixel, since the resolution level we are given for $F$ is the pixel. Therefore no larse 
moves may occur and when a point of the curve is close to an edge point, it is attracted by it. It will stabilize there if there is no conflict with the regularization process. But, normalizing $F$ implies that all forces have the same influence on the curve whatever their original magnitude is. This is not a major problem, once the edges are detected we make a treshold keeping only the points that are very likely to be real contours, and the curve find the equilibrium at local minima of the potential, which are edge points.

- space discretization: The force $F$ is known only on a discrete grid, corresponding to the image, and therefore, there can be a zero crossing without any zero in the grid. This means that in the best case, a point always oscillates between the pixels neighboring the minimum. This problem is simply solved by bilinear interpolation of $F$ at non integer positions. Thus we have a continuous definition of $F$ and equilibrium points correspond to the zeros of $F$.

Remark that even if the initial equation has been changed, the curve is still attached to minimize the original potential and energy.

We give below examples of results applying this method, first to a drawn line and then to medical images. In figure (2) image is a slice from a 3D MR image in the heart area. We want to extract the left ventricle. We use here the $3 \mathrm{D}$ edge detector obtained by generalization of the $2 \mathrm{D}$ Canny-Deriche filter [10]. We give in comparison exemples of what happens when we do not normalize the image forces (figure 3 ). If the time step is too large, the force $\tau F\left(v^{t-1}\right)$ is too large and, beginning from the initial estimation in figure (2). give instabilities such that points that are slightly on one side of a contour are moved far away on the other side.

\subsection{The balloon Model}

To make the snake find its way; an initial guess of the contour has to be provided manually. This has many consequences on the evolution of the curve (see figure 4). 
- If the curve is not close enough to an edge, it is not attracted by it.

- If the curve is not submitted to any force (which will happen in a constant intensity area), it reduces itself to a straight line or a single point. This is, because the curve behaves like a one pixel length physical spring.

Suppose we have an image of a black rectangle on a white background and a curve is placed inside the rectangle. Even though we have a perfect edge detection, the curve vanishes. If a point is close enough to an edge point, it is attracted by it, and neighboring curve points will also stick to the edge. If there are enough of such points, the rest of the curve follows the edge step by step. On the contrary, if the initial curve is surrounding the rectangle, even if it is far from the edges, by its natural evolution, it will eventually match the desired rectangle.

Let us also note that, due to noise, some isolated points are maxima of the gradient and can stop the curve when it passes by (see figure 5).

All these remarks suggest we add another force which makes the contour have a more dynamic behavior. We now consider our curve as a "balloon" (in 2D) that we inflate. From an initial oriented curve we add to the previous forces, a pressure force pushing outside as if we were introducing air inside. The force:

$$
F_{p}=k_{1} \vec{n}(s)
$$

is added to $F$, where $\vec{n}(s)$ is the normal unitary vector to the curve at point $v(s)$ and $k_{1}$ is the amplitude of this force (if we change the sign of $k_{1}$ or the orientation of the curve, it will have an effect of deflation instead of inflation). The curve expands and it is attracted and stopped by edges as before. But since there is a pressure force, if the edge is too weak the curve can pass through this edge, if it is a singularity with regard to the rest of the curve being inflated. In the gradient image of the rectangle above, we have removed some edges and added some spurious ones to illustrate 
those remarks. Starting from the same small curve as in the previous examples and obtain the whole rectangle (see figure 6). When the curve passes by a noise point in the rectangle image, it sticks to the point. But, since on its two sides the curve is expanding, the noise point becomes a singular point of the curve and is removed by the regularization effect after few iterations. When the balloon reaches an equilibrium, the points which stick to edges are slightly outside of the real contour since the edge force has to be in equilibrium with the inflation and regularization forces. We can then reduce the inflation force to localize the position of the curve.

\subsection{Accounting for previous local edge detection}

We want to account for a previous local edge detection, obtained for instance with a Canny-Deriche edge detector $[4,7,10]$. To do this, we define the attraction forces by simulating a potential defined by convolving the binary edge image with a Gaussian impulse response. This can be used either as the only image forces or together with an intensity-gradient image to enforce the detected edges. This is useful when the detected edges are broken into small segments which are not linked together. Using energy-minimizing curves in this case is a way to close contours. For example if we use a high threshold in order to keep only the points that are very likely to be real contours, the curve both closes and smooths the contour. We used this for example in the images of the rectangle (figure 6).

\subsection{Optimizing elasticity and rigidity coefficients}

The coefficients of elasticity and rigidity have a great importance in the behavior of the curve along time iterations. If $w_{1}$ and $w_{2}$ are around unity, the internal energy $E_{\text {int }}$ has a major influence and the image forces have small effect. In this case the curve is only regularized.

A correct choice for parameters is guided by numerical analysis considerations. We wish that the coefficients of the rigidity matrix have all the same order of magnitude. We obtain good results 
when the parameters are of the order of $h$ for $w_{1}$ and $h^{3}$ for $w_{2}$ where $h$ is the space discretization step.

\section{Numerical Solution by Finite Element Method}

As in the previous section we consider the evolution equation:

$$
\left\{\begin{array}{l}
\frac{\partial v(s, t)}{\partial t}-\frac{\partial}{\partial s}\left(w_{1}(s) \frac{\partial v(s, t)}{\partial s}\right)+\frac{\partial^{2}}{\partial s^{2}}\left(w_{2}(s) \frac{\partial^{2} v(s, t)}{\partial s^{2}}\right)=-\nabla P(v(s, t)) \\
v(s, 0)=v_{0}(s) \text { initial curve } \\
+ \text { Boundary conditions. }
\end{array}\right.
$$

To simplify the notations, we consider the same equation as (4) with null boundary conditions (this is done by a simple change of variables).

As the variables $s$ and $t$ are independent hence, the discretization of the equation (4) can be done separatly in space and time.

We solve this last equation using a Finite Elements Method (FEM). The main idea of the FEM is to approximate over the Sobolev space $H_{0}^{2}(\Omega)$ the solution of the associated variational problem: find $v \in H_{0}^{2}(\Omega)$ such that

$$
a(v, \varphi)=L(\varphi) \quad \forall \varphi \in H_{0}^{2}(\Omega)
$$

where $L$ is not supposed to depend on $v$ (see appendix for details). There exists a unique solution to this equation, since the bilinear form $a(\varphi, \psi)$ is symmetric and positive definite as soon as $w_{1}(s)$ and $w_{2}(s)>0$

A well-known approach for approximating such problems is Galerkin's method, which consists in defining a similar problem, called discrete problem, over a finite dimensional subspace $V_{h}$ of the Sobolev space $H_{0}^{2}(\Omega)$. The associated disrete problem for (5) is: find $v_{h} \in V_{h}$ such that

$$
a\left(v_{h}, u_{h}\right)=L\left(u_{h}\right) \quad \forall u_{h} \in V_{h}
$$


The finite element method is characterized by three aspects in the construction of the space $V_{h}$ :

- A tessellation is established over the set $[0,1]$, such that $[0,1]=\bigcup_{i=1}^{N}[i h,(i+1) h]$, where $N$ is the number of discretization points.

- The functions $v_{h} \in V_{h}$ are piecewise polynomials, in the sense that for each $K_{i}=[i h,(i+1) h]$ the space $P_{K_{i}}=\left\{v_{h \mid K_{i}}, \quad v_{h} \in V_{h}\right\}$ consists of polynomials.

- There exists a basis in the space $V_{h}$ whose functions have small support.

Given a number $N \geq 1$, we set $h=\frac{1}{N+1}$ and consider a uniform subdivision of $[0,1]$ of step size $h$, composed of the nodes $x_{i}=i h \quad 0 \leq i \leq N+1$.

Since the variational problem (5) is approximated over the space $H_{0}^{2}([0,1])$, the space $V_{h}$ must satisfies $V_{h} \subseteq \mathcal{C}^{1} \cap H_{0}^{2}\left(\{0,1)\right.$ (see for details $[5 j)$. The subspace $V_{h}$ is then defined by:

$$
V_{h}=\left\{v \leq \mathcal{C}^{1}([0,1]), v_{!\left(x_{i}, x_{i+1}\right]} \in P_{3}\left(\left[x_{i}, x_{i+1}\right]\right) 0 \leq i \leq N\right\}
$$

where $P_{k}(I)$ is the vector space of the restrictions to an interval $I \tau R$ of the polynomials whose degree is less than $k$, and $v_{i} I$ is the restriction of the function $v$ to the subset $I$. The space $P_{3}$ have been choosen since, a polynomial $p \in P_{3}$ is uniquely determined by its values and the values of its derivative at two distincts points.

The basis functions of the finite element subspace $V_{h}$ are $\varphi_{i}$ and $\psi_{i}, 1 \leq i \leq N$ defined in a unique way by:

$$
\begin{aligned}
& \varphi_{i}\left(x_{j}\right)=\delta_{i j}, \quad \varphi_{i}^{\prime}\left(x_{j}\right)=0 \quad 1 \leq j \leq N \\
& \psi_{i}\left(x_{j}\right)=0, \quad \psi_{i}^{\prime}\left(x_{j}\right)=\delta_{i j} \quad 1 \leq j \leq N
\end{aligned}
$$

where:

$$
\delta_{i j}= \begin{cases}1 & \text { if } i=j \\ 0 & \text { otherwise }\end{cases}
$$


(see appendix for the expression of $\varphi_{i}$ and $\psi_{i}$ ).

Thus $\nabla u_{h} \sqsubseteq V_{h}$ we have the identity:

$$
v_{h}=\sum_{i=1}^{N} v_{h}\left(x_{i}\right) \varphi_{i}+\sum_{i=1}^{N} v_{h}^{\prime}\left(x_{i}\right) \psi_{i}
$$

yielding an analytic expression of the solution over the set $[0,1]$. Finally the solution of the discrete problem in space (6) is equivalent to a solution of the linear system:

$$
A \cdot V=L
$$

where the matrix $A$ is symmetric, definite positive and heptadiagonale. The reader can find the details in the appendix.

\subsection{Discretization of the evolution problem}

After we discretized the problem (4) in space, we discretize the equation (13) using finite differences in time. This leads to:

$$
\left\{\begin{array}{l}
\frac{V^{t}-V^{t-1}}{\tau}+A \cdot V^{t}=L_{V^{t-1}} \\
V^{0}=v_{0} \text { initial estimation. }
\end{array}\right.
$$

where $\tau$ is the time step. Equation (10) can be written similarly to the finite differences formulation (3):

$$
(I d+\tau \cdot A) \cdot V^{t}=V^{-t-1}+\tau L_{V^{\prime-1}}
$$

We have to solve a linear system $M \cdot I=N$ at each time step, for which the matrix $M=(I d-T A)$ is banded symmetric positive ( $M$ is heptadiagonal). Since $M$ does not depend on $t$, one must inverse it only once using a Cholesky factorization if the coefficients $w_{i}$ remains constants. 


\subsection{The balloon model}

As described in section 3.3, the equation becomes:

$$
\left\{\begin{array}{l}
\frac{\partial v}{\partial t}-\frac{\partial}{\partial s}\left(w_{1}(s) \frac{\partial v}{\partial s}\right)+\frac{\partial^{2}}{\partial s^{2}}\left(w_{2}(s) \frac{\partial^{2} v}{\partial s^{2}}\right)=-k \frac{\nabla P}{\|\nabla P\|}(v)+k_{1} \vec{n}(s, t) \\
v_{0}(s)=v(s, 0)
\end{array}\right.
$$

where $\vec{n}(s)$ is the external normal vector at the point $v(s)$. So only the expression of vector $L$ is changed to take into account the added force. Coefficients $k_{1}$ and $k$ are chosen such that they are of the same order, (less than one pixel size), with $k$ slightly larger than $k_{1}$ to allow an edge point to stop the inflation force. See results on figures 7 and 8 .

\section{Performances and complexity analysis}

If we compare our results with those obtained using a finite differences method (FDM) (as in [6]), we find out that, as expected, the finite elements method requires less points in the curve discretization and gives more stable results. This is due to the computation of the vector $L$ in equation (11) and in the nature of the method. In the FDM, we follow the evolution of a set of points. In the FEM, we actually deform the curve which is between two points of the grid, so the image forces between two points are also considered. The computation of vector $L$ is made by numerical integration, interpolating along the interval $[(i-1) h,(i+1) h]$ for each node $x_{i}=i h$ of the subdivision. The numerical integration is made at the pixel size so that no information is lost. This FEM gives also a faster convergence to an equilibrium.

Since the methods leads to the solution of a linear system (10) or (3), algorithmic complexity can be compared by exanuining the associated linear systems.

Let set $N$, the number of the discretization points of the intervals $[0,1$. For the FEM, the $A$ is a $2 N \times 2 N$ heptadiagonal array, while it is only $N \leqslant N$ pentadiagonal for the FDM. But in FDM 
the number of points $N$ must be at least equal to the length $l$, in pixels, of the initial guess, not to miss too much informations. This leads to the solution of a bigger linear system.

On the contrary, for the finite element method the number of points $N$ is typically of the order

of $l ; 6$. Thus the algorithmic complexity for the FEM, in this case is $\frac{36}{4}=9$ times cheaper than with the FDM.

This advantage comes in addition to the previously mentionned ones: more accurate computation of the positions and all derivatives at any point of the curve.

\section{$63 \mathrm{D}$ reconstruction and temporal tracking}

With our method, we experimented the reconstruction of a $3 \mathrm{D}$ surface by initializing a balloon model in an intermediate cross section, and by propagating the result to neighboring cross-sections, initializing in a cross section the curve with the result obtained in the previously processed connected section (as in [1] where the curves were extracted by hand, on each slice, using an image of edges). We made a first approach to $3 \mathrm{D}$ reconstruction by extracting the contour slice by slice. Figures 9 and 10 show the reconstruction of the left and right ventricles. This reconstruction is almost automatic. Indeed, when the contour undergoes a big change from one slice to the next, the initial curve in that slice may have to be redefined in order to obtain a good contour, problem which can be avoided by adding interpolated slices when necessary. We remark that this problem never arises when the image solution is the same in the three axes.

\section{$7 \quad$ Future directions}

The next step in our research is to follow the deformation of this surface in time. This can be done either slice by slice or globally by generalizing this approach to a 3D surface model which would be 
a real balloon since the active contour model is a particular case of the deformable models of [12]. We have generalized this work to a $3 \mathrm{D}$ surface model and some interesting and promising results are expected soon.

We can add internal forces to control the deformation for following the contours. This is the case if we have a physical model of the desired object (for example, following the deformation of a ventricle during a cardiac cycle), or for making the curve expand or contract from the initial data using some knowledge of the deformation properties.

Another direction of our research is the elastic matching of extracted features to an atlas, which is related to [2]. A deformation between the pattern and the object is allowed. Thus we deform a curve to best match the pattern using some of the measure distorsion to achieve the deformation, such as the area between the two curves. This was also studied in [13] with simple geometric shapes as templates which are deformed to match the inage.

\section{Conclusion}

We have presented a model of deformation which can solve some of the problems encountered with the "snake" model of [9]. We have modified the definition of external forces deriving from the gradient of the image to obtain more stable results, and introduced a pressure force which make the curve model behave like a balloon. This allows to give an initial guess of the curve which is far from the desired solution. We have presented a finite elements method to solve a model of deformation of a balloon similar to the "snake" model. There are many advantages versus finite differences as, more stability, faster convergence and coarser parameterization of the curve. We showed promising results of our model on MR (magnetic resonance) images, to extract features like the contour of a heart ventricle on $2 \mathrm{D}$ slices. Using a series of such contours in successive cross 
sections, we made a $3 \mathrm{D}$ reconstruction of the inside surface of the ventricles. This method has been tested for several applications in medical image analysis and it is actually generalized to a full 3D model of surfaces. To segment more efficiently $3 D$ images. This generalization is described in a companion paper.

\section{Appendix: details on the numerical solution}

\section{A. Variational formulation}

Let $\varphi \in H_{0}^{2}([0,1])$ be a smooth function. If $v$ is a solution of equation (4), the associated variationnal formulation is:

$\int_{0}^{1} \frac{\partial v(s, t)}{\partial t} \varphi(s) d s-\int_{0}^{1} \frac{\partial}{\partial s}\left(w_{1}(s) \frac{\partial v(s, t)}{\partial s}\right) \varphi(s) d s+\int_{0}^{1} \frac{\partial^{2}}{\partial s^{2}}\left(w_{2}(s) \frac{\partial^{2} v(s, t)}{\partial s^{2}}\right) \varphi(s) d s=-\int_{0}^{1} \nabla P(v(s, t)) \varphi(s) d s$

We remark that the variables $s$ and $t$ are independents, we can separate them (for more details see

[5]). We obtain, using the Green's formula:

$\frac{d}{d t} \int_{0}^{1} v(s, t) \varphi(s) d s+\int_{0}^{1} w_{1}(s) \frac{\partial v(s, t)}{\partial s} \varphi^{\prime}(s) d s+\int_{0}^{1} w_{2}(s) \frac{\partial^{2} v(s, t)}{\partial s^{2}} \varphi^{\prime \prime}(s) d s=-\int_{0}^{1} \nabla P(v(s, t)) \varphi(s) d s$

Let us set:

$$
a(\varphi, \psi)=\int_{0}^{1} w_{1}(s) \varphi^{\prime}(s) \psi^{\prime}(s) d s+\int_{0}^{1} w_{2}(s) \varphi^{\prime \prime}(s) \psi^{\prime \prime}(s) d s
$$

and

$$
L(\psi)=-\int_{0}^{1} \nabla P(v(s, t)) \psi(s) d s
$$

This leads us to a new formulation of the problem: given $v_{0} \in L^{2}(\Omega)$ and $\nabla P \in L^{2}\left(0, T, L^{2}(\Omega)\right)$, find a function $v \in L^{2}\left(0, T, H_{0}^{2}(\Omega)\right) \cap \mathcal{C}^{1}\left(0, T, L^{2}(\Omega)\right)$ satisfying:

$$
\left\{\begin{array}{l}
\frac{d}{d t}(v(t), \psi)+a(v(t), \psi)=L_{v}(\psi) \forall \psi \in H_{0}^{2}(\Omega) \\
v(0)=v_{0} \\
w_{1}(s), w_{2}(s) \in L^{\infty}(\Omega), w_{1}(s) \text { and } w_{2}(s) \geq \alpha>0
\end{array}\right.
$$


Sinc the variables $s$ and $t$ are independent, we can solve the equation (13) in two steps: first solve the static equation: find $v \in H_{0}^{2}(\Omega)$ such that

$$
a(v, \varphi)=L(\varphi) \quad \forall \varphi \in H_{0}^{2}(\Omega)
$$

where $L$ is not supposed depending on $v$ (we remind to the reader that there exists a unique solution to this equation, since the bilinear form $a(\varphi, \psi)$ is symmetric and positive definite as soon as $w_{1}(s)$ and $\left.w_{2}(s)>0\right)$, and then solve the evolution equation (13).

\section{B. $V_{h}$ basis functions}

Using relations ( $(\tau)$ and $(8)$ we obtain:

$$
\varphi_{j}(x)= \begin{cases}-\frac{2}{h^{3}} x^{3}-\frac{3}{h^{2}}(1-2 j) x^{2}+\frac{6 j}{h}(1-j) x+1+j^{2}(2 j-3) & \text { if } x \in\left[x_{j-1}, x_{j}\right] \\ \frac{2}{h^{3}} x^{3}-\frac{3}{h^{2}}(1+2 j) x^{2}+\frac{6 j}{h}(1+j) x+1-j^{2}(2 j+3) & \text { if } x \in\left[x_{j}, x_{j+1}\right] \\ 0 & \text { elsewhere. }\end{cases}
$$

and

$$
\psi_{j}(x)= \begin{cases}\frac{1}{h^{2}} x^{3}+\frac{1}{h}(2-3 j) x^{2}+\left(3 j^{2}-4 j+1\right) x-j(j-1)^{2} & \text { if } x \in\left[x_{j-1}, x_{j}\right] \\ \frac{1}{h^{2}} x^{3}-\frac{1}{h}(2+3 j) x^{2}+\left(3 j^{2}+4 j+1\right) x-j(j+1)^{2} & \text { if } x \in\left[x_{j}, x_{j+1}\right] \\ 0 & \text { elsewhere. }\end{cases}
$$

Thus $\forall v_{h} \in V_{h}$ we have the identity:

$$
v_{h}=\sum_{i=1}^{N} v_{h}\left(x_{i}\right) \varphi_{i}+\sum_{i=1}^{N} v_{h}^{\prime}\left(x_{i}\right) \psi_{i}
$$

\section{Discrete problem and linear system}

Rewritting the discrete problem (5) with the basis functions $\varphi_{i}$ and $\psi_{i}$, give us the equations:

$$
\forall j=1, \ldots, N\left\{\begin{array}{l}
a\left(v_{h}, \varphi_{j}\right)=L\left(\varphi_{j}\right) \\
a\left(v_{h}, \psi_{j}\right)=L\left(\psi_{j}\right)
\end{array}\right.
$$


and using the identity (9), we have:

$$
\forall j=1, \ldots, N\left\{\begin{array}{l}
\sum_{i=1}^{N} v_{h}\left(x_{i}\right) a\left(\varphi_{i}, \varphi_{j}\right)+\sum_{i=1}^{N} v_{h}^{\prime}\left(x_{i}\right) a\left(\psi_{i}, \varphi_{j}\right)=L\left(\varphi_{j}\right) \\
\sum_{i=1}^{N} v_{h}\left(x_{i}\right) a\left(\varphi_{i}, \psi_{j}\right)+\sum_{i=1}^{N} v_{h}^{\prime}\left(x_{i}\right) a\left(\psi_{i}, \psi_{j}\right)=L\left(\psi_{j}\right)
\end{array}\right.
$$

The equation (17) is a linear system, where the unknowns are $v_{h}\left(x_{i}\right)$ and $v_{h}^{\prime}\left(x_{i}\right)$ for $i=1, \ldots, N$.

Finally the solution of the discrete problem (6) is equivalent to a solution of the linear system:

$$
A \cdot V=L
$$

where:

- $A$ is a tridiagonal blocs array $A=\left(A_{i j}\right)_{i, j=1, \ldots, N}$ with:

$$
A_{i j}=\left(\begin{array}{cc}
a\left(\varphi_{i}, \varphi_{j}\right) & a\left(\psi_{i}, \varphi_{j}\right) \\
a\left(\varphi_{i}, \psi_{j}\right) & a\left(\psi_{i}, \psi_{j}\right)
\end{array}\right)
$$

the $A_{i j}$ array elements depend on the elasticity and rigidity coefficients.

$-V=\left(v_{h}\left(x_{1}\right), v_{h}^{\prime}\left(x_{1}\right), \ldots, v_{h}\left(x_{N}\right), v_{h}^{\prime}\left(x_{N}\right)\right)^{T}$

- and $L=\left(L\left(\varphi_{1}\right), L\left(\psi_{1}\right), \ldots, L\left(\varphi_{N}\right), L\left(\psi_{N}\right)\right)^{T}$.

\section{Computation of the vector $L$}

The computation of $L=\left(L\left(\varphi_{1}\right), L\left(\psi_{1}\right), \ldots, L\left(\varphi_{i}\right), L\left(\psi_{N}\right)\right)^{T}$ depends upon $P=-|\nabla I(x, y)|^{2}$ which is known only at integer values. Thus the integrals

$$
\int_{0}^{1} \nabla P(v(s, t)) e_{i}(s) d s
$$

where $e_{i}=\left(\varphi_{i}, \psi_{i}\right)$, will be computed with a numerical integration. The formula used is:

$$
\int_{a}^{b} \phi(x) d x \simeq(b-a) \phi\left(\frac{a+b}{2}\right)
$$

This formula suppose that the gradient of the potential function is known at any point $(x, y)$, but only the values at integer points are known. Consequently at each point $(x, y)$, we use a linear 
interpolation of the function $\nabla P$ depending on the four neighbors points. We obtain:

$$
L\left(\varphi_{i}\right)=-\frac{h}{2}\left(v^{\prime}\left(\frac{(2 i-1) h}{2}, t\right) \mathcal{F}\left(v\left(\frac{(2 i-1) h}{2}, t\right)\right)+v^{\prime}\left(\frac{(2 i+1) h}{2}, t\right) \mathcal{F}\left(v\left(\frac{(2 i+1) h}{2}, t\right)\right)\right)
$$

and

$$
L\left(\psi_{i}\right)=-\frac{h^{2}}{8}\left(-v^{\prime}\left(\frac{(2 i-1) h}{2}, t\right) \mathcal{F}\left(v\left(\frac{(2 i-1) h}{2}, t\right)\right)+v^{\prime}\left(\frac{(2 i+1) h}{2}, t\right) \mathcal{F}\left(v\left(\frac{(2 i+1) h}{2}, t\right)\right)\right)
$$

where $\mathcal{F}(v(s, t))$ is the interpolated value of $\nabla P-F_{p}$ at the point $v(s, t)$.

In fact as we pointed out in section (5), the numerical integration formula of equation (18) is in fact more precise to take into account the information at all pixels that are in the corresponding segment on the image.

\section{References}

(1) N. Ayache, J.D. Boissonnat, E. Brunet, L. Cohen, J.P. Chièze, B. Geiger, O. Monga, J.M. Rocchisani, and P. Sander. Building highly structured volume representations in $3 \mathrm{~d}$ medical images. In Computer Aided Radiology, 1989. Berlin, West-Germany.

[2] Ruzena Bajcsy and Stane Kovacic. Multiresolution elastic matching. Computer Vision, Graphics, and Image Processing, 46:1-21, 1989.

[3: Andrew Blake and Andrew Zisserman. Visual Reconstruction. The MIT Press, 1987.

[4] John Canny. A computational approach to edge detection. IEEE Transactions on Pattern Analysis and Machine Intelligence, PAMI-8(6):679-698, November 1986.

[5] P. G. Ciarlet. The finite element methods for elliptic problems. NORTH-HOLLAND, 1987.

[6] Laurent D. Cohen. On active contours models. Technical Report 1075, INRIA, August 1989. 
[i] Rachid Deriche. Using canny's criteria to derive a recursively implemented optimal edge detector. International Journal of Computer Vision, pages 167-187, 1987.

(8) Pascal Fua and Yvan G. Leclerc. Model driven edge detection. In DARPA Image Understanding Workshop, 1988.

[9] Michael Kass, Andrew Witkin, and Demetri Terzopoulos. Snakes: Active contour models. International Journal of Computer Vision, 1:321-331, 1987.

[10! O. Monga and R. Deriche. $3 \mathrm{~d}$ edge detection using recursive filtering. application to scanner images. In IEEE Computer Society C'onference on Vision and Pattern Recognition, San Diego, June 1989.

[11] Demetri Terzopoulos. On matching deformable models to images. Technical Report 60, Schlumberger Palo Alto Research, November 1986.

[12] Demetri Terzopoulos. The computation of visible-surface representations. IEEE Transactions on Pattern Analysis and Machine Intelligence, PAMI-10(4):41i-438, July 1988.

13! A.L. Yuille. D.S. Cohen, and P.W. Hallinan. Feature extraction from faces using deformable templates. In Proceedings of Computer Vision and Pattern Recognition, San Diego, June 1989. 


\section{Figures}

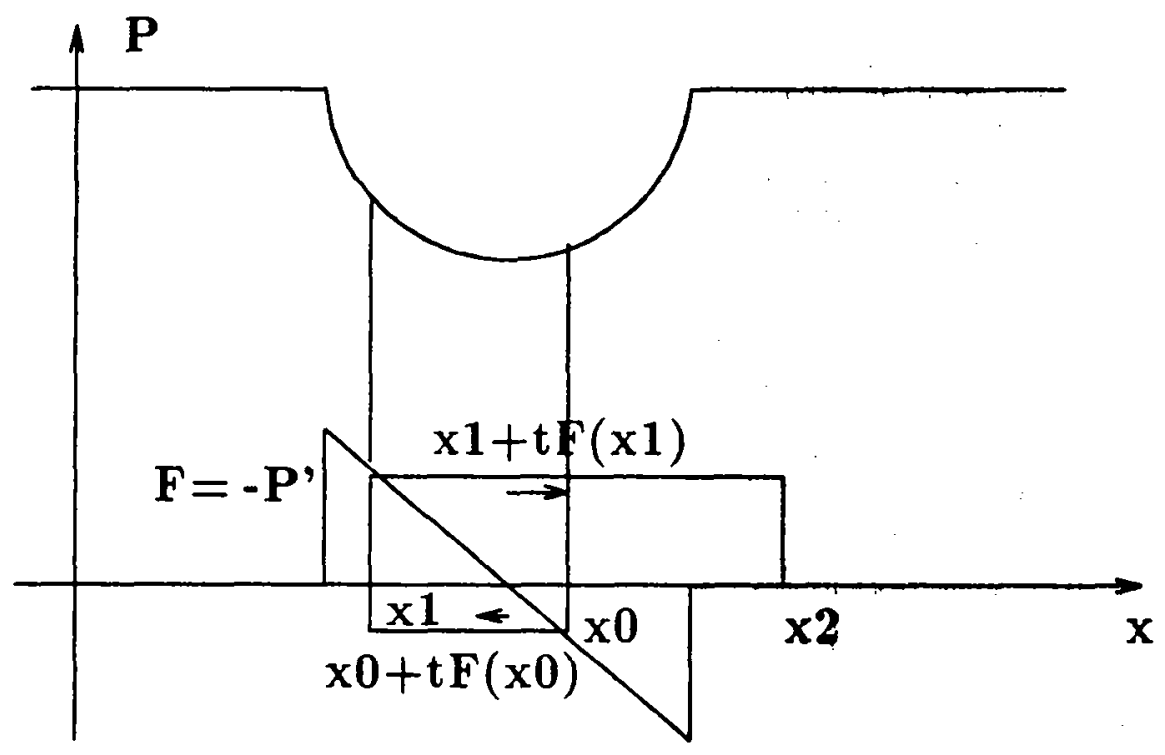

Figure 1: instability due to time discretization. Starting from $x 0, t F(x 0)$ is too large and we go away from the good minimum to $x 2$ which is also an equilibrium. 

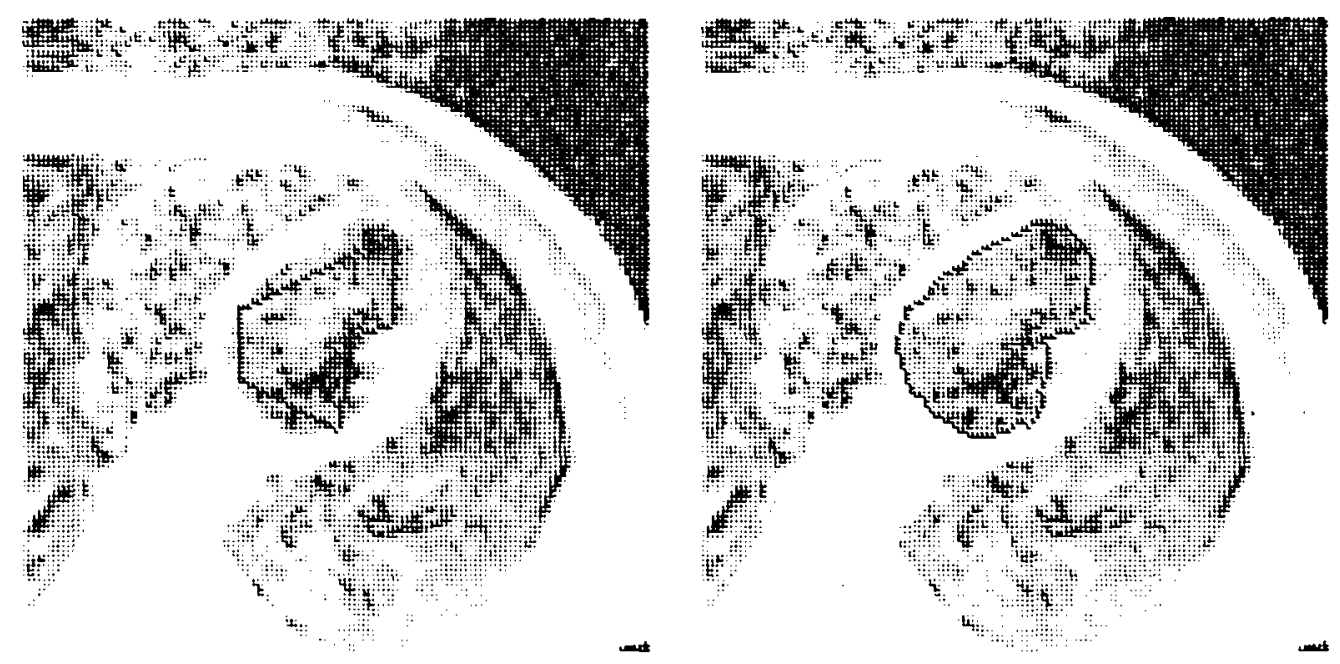

4

Figure 2: We detect here the contour of the left ventricle in a MR image of the heart. On the left, the initial curve is given by the operator with only six points. On the right the stable curve obtained after several iterations.
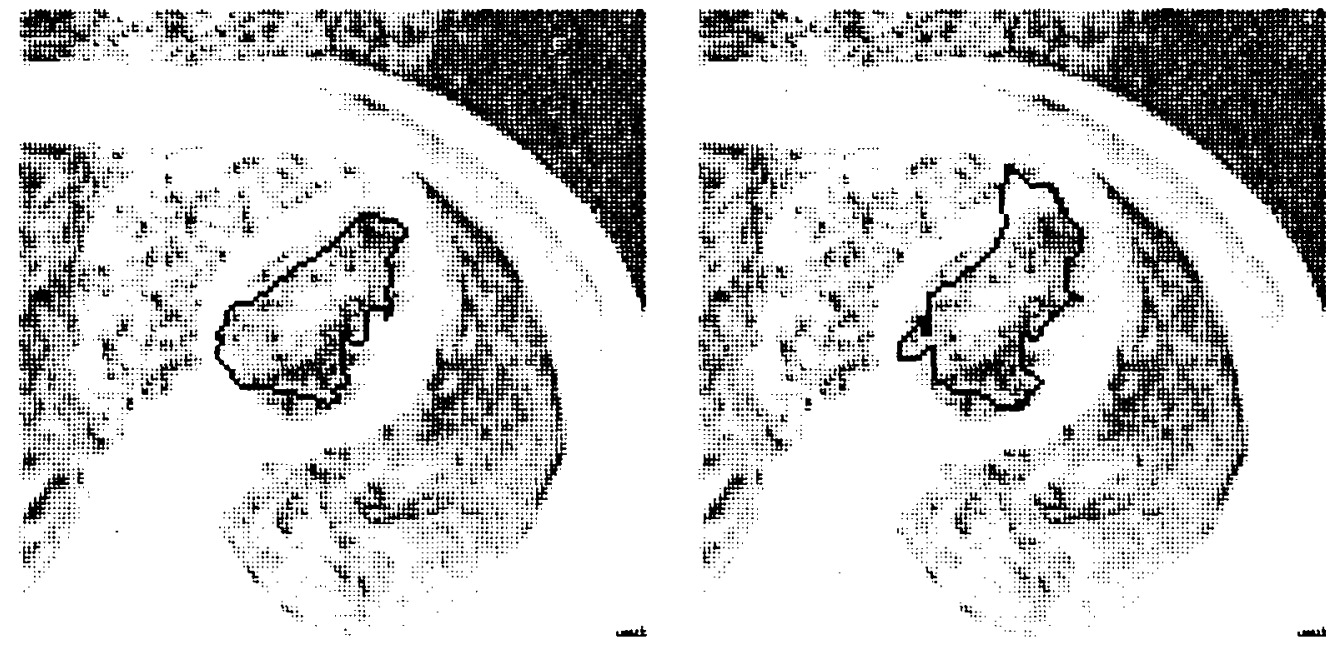

Figure 3: Example of instability when the image forces are not normalized. These are two successive iterations when the initial curve is close to the solution. 

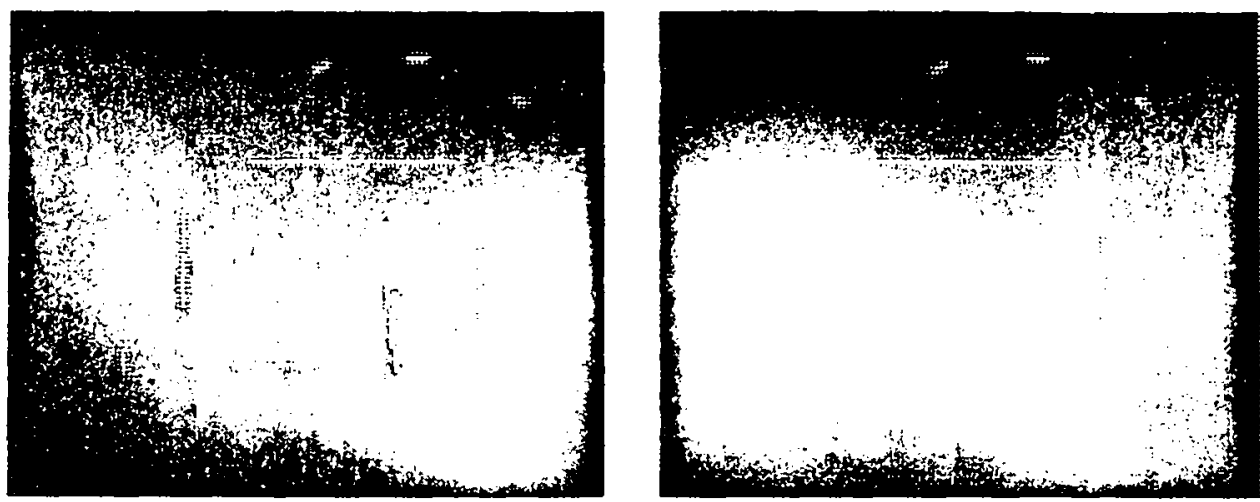

Figure 4: Problem due to distant edges: the initial curve ( $\Lambda$ curve, on bottom right) is too far from the desired shape (broken rectangle), the curve tends to collapse to a straight line.
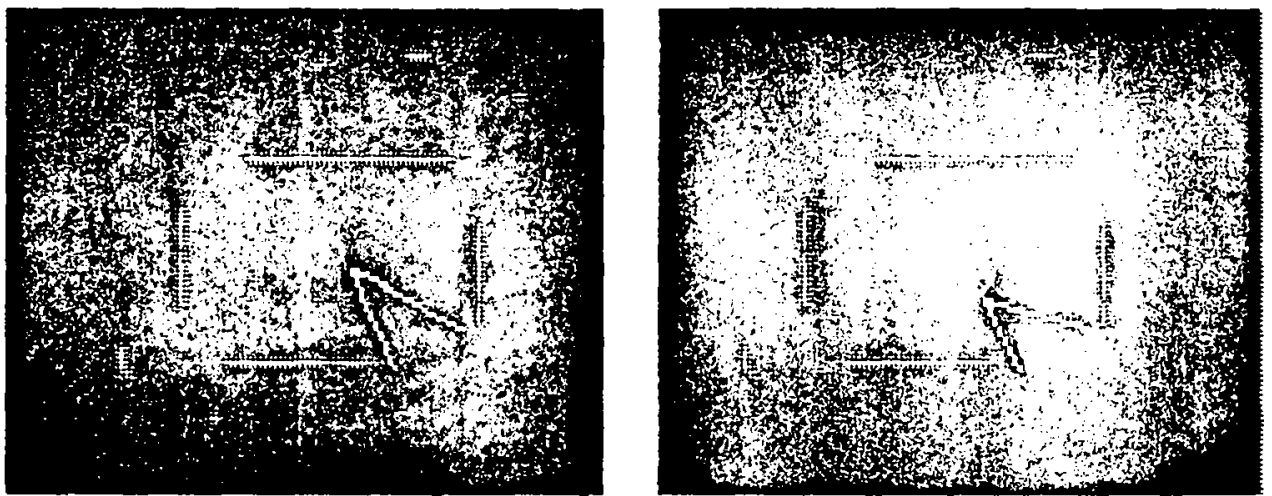

Figure 5: Problem due to spurious edges: the initial curve ( $\Lambda$ curve, on bottom right) is "trapped" by a single spurious isolated edge point.
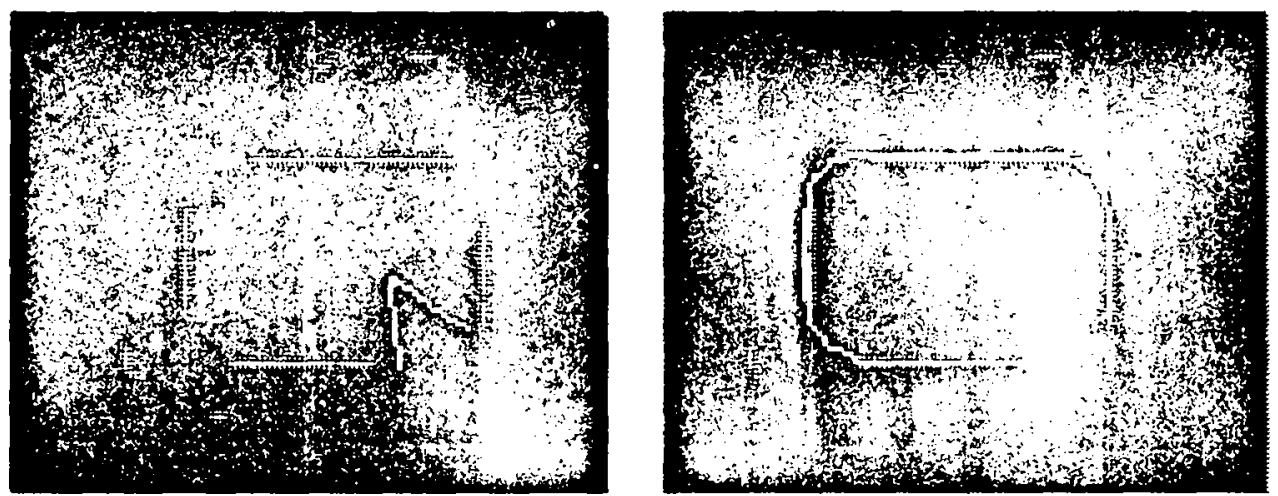

Figure 6: Advantage of the balloon model: the initial curve neither collapse nor gets trapped by spurious isolated edges points. It, robustly converges toward the desired rectangle shape. 

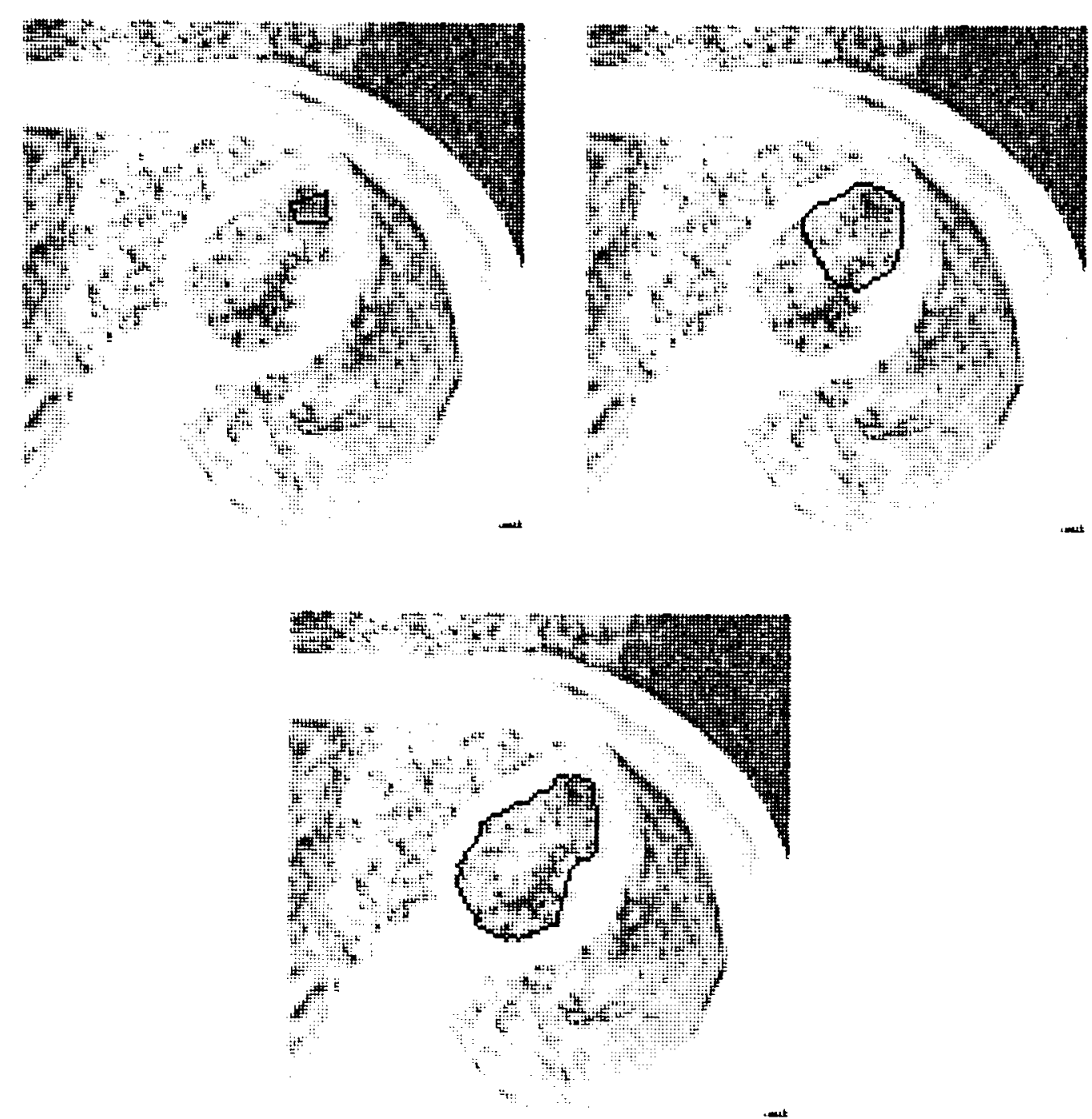

Figure 7: Illustration of the robustness of the balloon model: the final result can be achieved from almost any initial curve given within the interior of the ventricle (see also the next figure) 

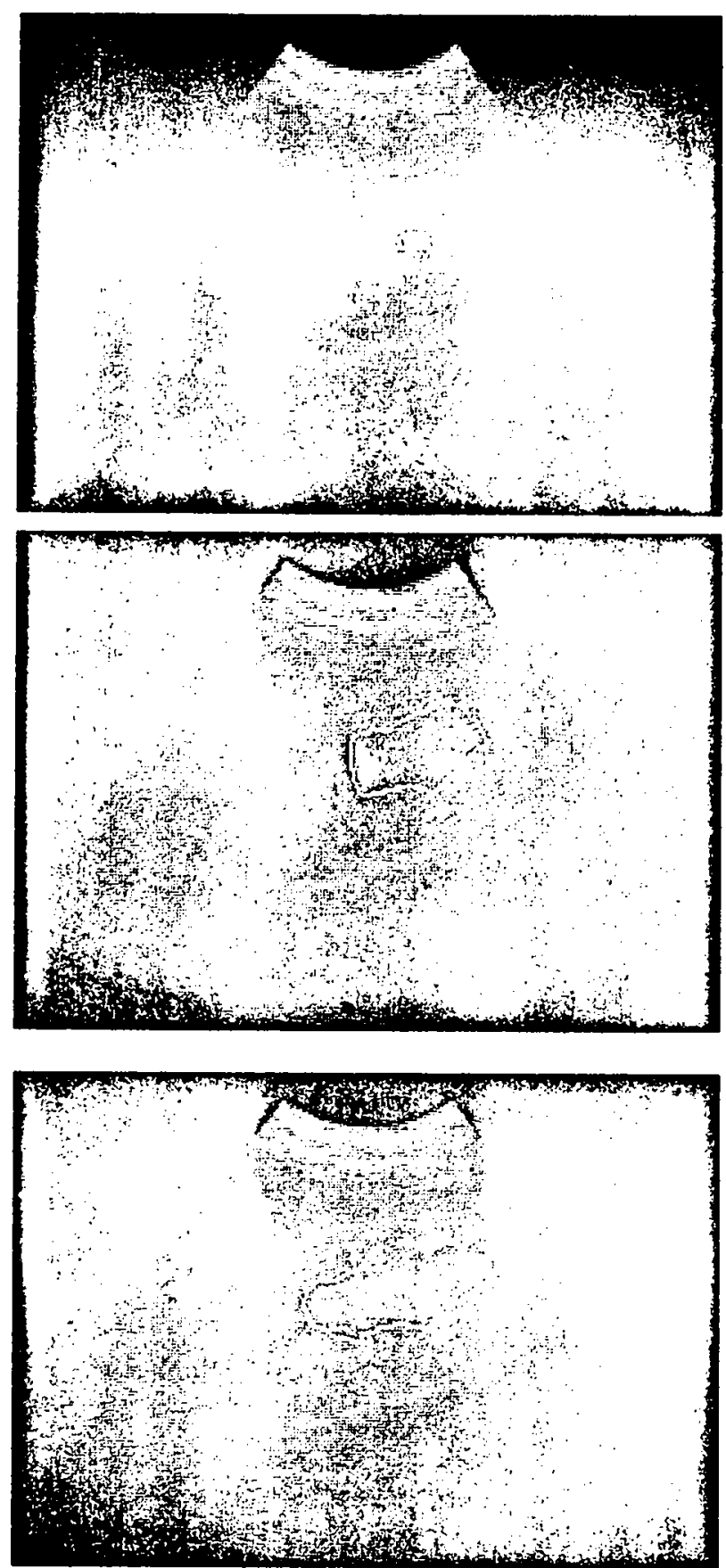

Figure 8: Echographic images. Evolution of the balloon curve to detect the left ventricle 

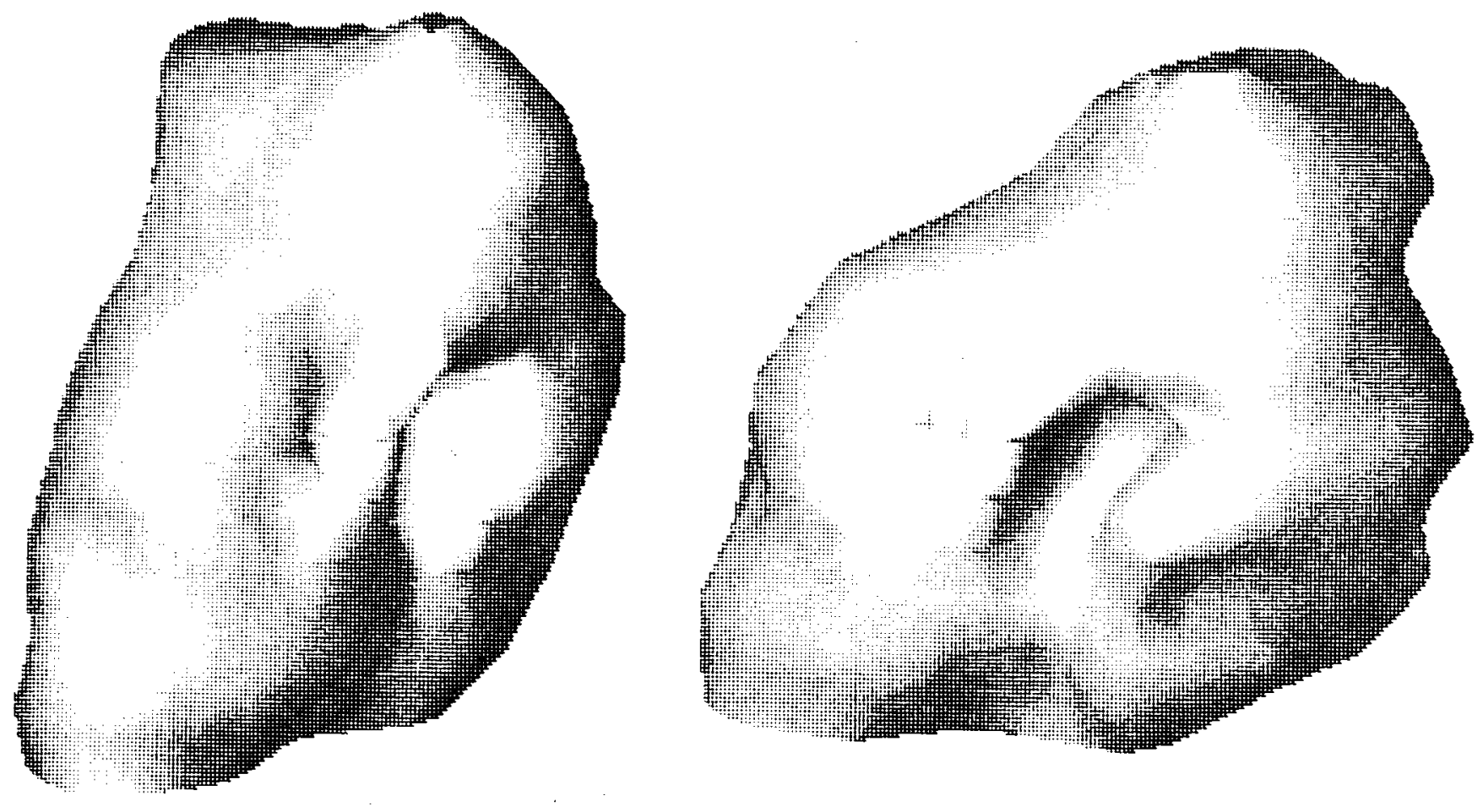

Figure 9: two views of the reconstructed inside cavity of the left ventricle 


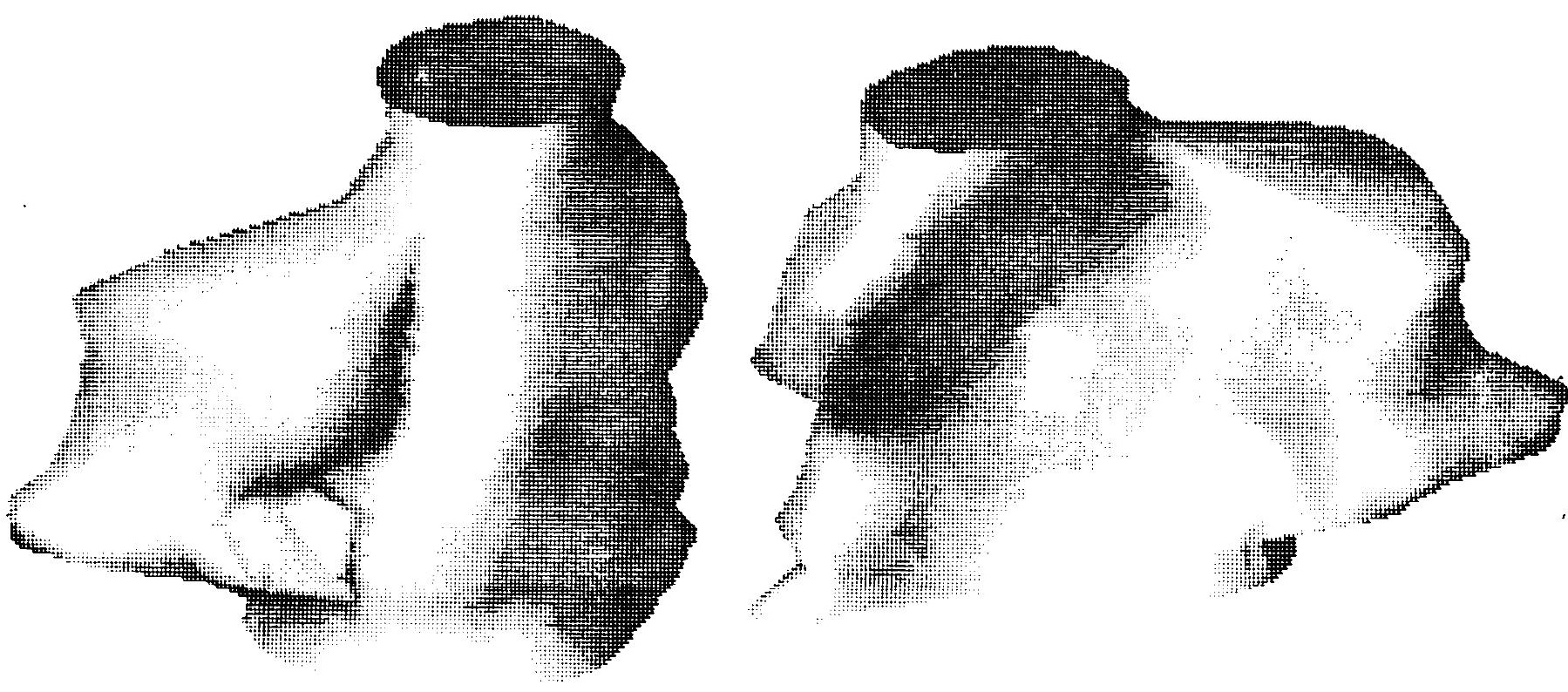

Figure 10: two views of the reconstructed inside cavity of the right ventricle 
Imprimé en France

PInstivus National de Rechesche en Informatique et en Automatique 
ISSN 0249-6399 